\title{
Femoral hernia appendix causing small intestinal obstruction
}

\author{
J.P. Wyatt and J.S. Varma
}

Department of Surgery, Alexander Donald Building, Western General Hospital, Crewe Road, Edinburgh, UK

\begin{abstract}
Summary: An 88 year old woman presented with a painful, irreducible right femoral hernia and small intestinal obstruction. At laparotomy, some distal small intestine was found to have twisted around an uninflamed appendix which was acting as a 'band', with its tip fixed in the femoral hernia sac. Although the association between the appendix and femoral hernia is well recognized, the production of small intestinal obstruction by this particular mechanism has not been previously reported.
\end{abstract}

\section{Introduction}

Small intestinal obstruction secondary to a femoral hernia is seen relatively frequently in general surgical practice, usually caused by small intestine trapped in the hernial sac. In this case report we describe small intestinal obstruction resulting from a femoral hernia, but by a previously undescribed mechanism.

\section{Case report}

An 88 year old woman was admitted as an emergency with a 3 day history of central abdominal pain, constipation, nausea and abdominal distension. Her past medical history included an abdominal hysterectomy 15 years previously. On examination, she was clinically dehydrated, with a distended abdomen, maximally tender to the right of a lower midline scar and over an irreducible right femoral hernia. Plain abdominal X-rays showed multiple dilated loops of small intestine in keeping with the clinical diagnosis of an obstructed right femoral hernia. A nasogastric tube was passed, the patient was rehydrated with intravenous fluids and prepared for surgery.

The abdomen was opened through the lower midline scar, revealing an apparently uninflamed appendix lying under tension as a tight band with its tip fixed in the right femoral hernia sac. Distal small intestine had twisted around this unusual 'band' causing small intestinal obstruction, with

Correspondence: J.P. Wyatt, B.Med.Sci., M.B., Ch.B., Department of Surgery, Alexander Donald. Building, Western General Hospital, Edinburgh EH4 2XU, UK. Accepted: 30 July 1991 distended proximal bowel which was characterized by multiple diverticula. The small intestine distal to the obstruction appeared normal and once untwisted, the entire bowel appeared viable. The appendix was freed from the femoral hernia sac and both were excised. The femoral hernia was repaired and the abdomen closed. The patient made an uneventful recovery. Histopathology of the appendix confirmed it to be uninflamed.

\section{Discussion}

There is a well-recognized association between the vermiform appendix and femoral herniae (particularly those on the right side), usually discovered incidentally during hernia repair. Following a description in 1731 by De Garengeot ${ }^{1}$ there have been reports of appendicitis, appendiceal carcinoma and strangulation within femoral herniae. ${ }^{2,3}$ Whilst the occurrence of the appendix within a femoral hernia has been accurately predicted preoperatively, ${ }^{4}$ it is more common for it to be discovered only at operation, as in this study. Because of previous surgery the abdomen was opened by a lower midline incision providing excellent access.

The jejunal diverticula seen may have been incidental, although it might be postulated that they resulted from increased intraluminal pressure due to distal obstruction. However, this seems unlikely in view of the relatively short history.

In summary, this case report illustrates 2 points. Firstly, the unpredictably obscure way in which the appendix can produce intra-abdominal mischief; secondly, the advantage provided by a 'high' approach for femoral hernia complicated by intestinal obstruction. 


\section{Acknowledgements}

We thank Mr D.W. Hamer-Hodges for permission to report this case.

\section{References}

1. De Garengeot, R.J.C. Traité des operations de chirurgie, 2nd ed. Paris, Huart, 1731, pp.369-371.

2. Khatib, C.M. Strangulated femoral hernia containing acute gangrenous appendicitis: case report and review of the literature. Can J Surg 1987, 30: 50.

3. Spencer, R.F. et al. Unusual combined pathology in a femoral hernia. J R Coll Surg Edin 1984, 29: 255-256.

4. Gray, H.T. Lesions of the isolated appendix vermiformis in the hernial sac. $\mathrm{Br}$ Med J 1910, 2: 1142-1145. 